«Системні технології» 3 (128) 2020 «System technologies»

DOI 10.34185/1562-9945-3-128-2020-08

УДК 519.6

Ю.В. Бразалук, А.И. Губин, А.В. Давыдова, В.С. Дерий, Д.В. Евдокимов, Ю.А. Малая

\title{
МАТЕМАТИЧЕСКОЕ МОДЕЛИРОВАНИЕ БАЛЛОННЫХ СИСТЕМ ХРАНЕНИЯ И РЕГУЛИРОВАНИЯ РАСХОДА ГАЗА
}

Аннотаця. В работе рассмотрены процессы истечения газа через пористую вставку из баллона высокого давления, используемого для газоснабжения космического летательного аппарата в длительном полете. Для описания процесса фильтрации была использована математическая модель изотермической фильтрации газа. Применение асимптотического подхода позволило свести описание прочесса фильтрации к краевой задаче для обыкновенного дифференциального уравнения второго порядка, которую удалось решить аналитически. Показано, что в качестве управляющих параметров процесса истечения газа можно использовать температуру газа внутри баллона или давление газа на выходе пористой вставки. Результаты работы могут быть рекомендованы для использования в ракетно-космической технике и других областях, связанных с хранением сжатого газа.

Ключевые слова: сжатый газ, инертный газ, баллон высокого давления, температура газа, изотермическая фильтрация газа, асимптотический метод, аналитическое решение, космический летательный аппарат, длительный полет.

Введение. Многочисленные технические системы и производственные технологии предусматривают длительное хранение газов и их потребление в весьма малых количествах, но на протяжении длительного времени. Нередко такие системы используются в авиации и ракетнокосмической технике, где емкость, содержащую газ, приходится перевозить на движущемся транспортном средстве. Дальнейшее рассмотрение проведем на примере космических летательных аппаратов, в том числе искусственных спутников и орбитальных станций. Актуальность вопросов обеспечения надлежащего функционирования таких систем непосредственно определяет важность тематики настоящего исследования для современной науки и техники.

(C) Бразалук Ю.В., Губин А.И., Давыдова А.В., Дерий В.С., Евдокимов Д.В.,Малая Ю.А., 2020 80 
«Системні технології» 3 (128) 2020 «System technologies»

В современных космических летательных аппаратах зачастую возникает потребность использовать газообразные вещества как для обеспечения функционирования таковых аппаратов, так и с целью проведения научных исследований. Чаще всего, благодаря специфическим физико-химическим, теплофизическим и механическим свойствам в космических летательных аппаратах применяют инертные газы (аргон, ксенон, криптон, гелий). Благодаря специфике условий длительного космического полета (условия микрогравитации, трудноконтролируемый тепловой режим аппарата) проблема хранения и дозирования расхода газов при их использовании представляется весьма нетривиальной и требующей специальных теоретических и экспериментальных исследований. Настоящая работа, главным образом, посвящена разработке методик расчета условий хранения газов на борту космических летательных аппаратов и управления их расходом.

Постановка проблемы в общем виде и ее связь с важными научными и практическими задачами. В обычной наземной технике существует три основных способа обеспечения поставки газа для работы технических устройств: хранение газов под высоким давлением в специально спроектированных баллонах; применение газогенераторов; применение парогенераторов. В условиях космического полета, с учетом необходимости сохранять герметичность системы и не допускать в ней больших скачков давления, использование газогенераторов и парогенераторов представляется затруднительным. Поэтому предпочтение отдается баллонам со сжатым газом под достаточно высоким давлением. Традиционные системы дозирования расхода газа также плохо применимы в условиях космического полета. Как будет показано ниже, наиболее простым и приемлемым решением в данном случае является управление состоянием газа и расходом его в соответствии с потребностями функционирования космической системы при помощи регулирования температурного режима баллона, содержащего газ. Вторым элементом управления расходом газа, обеспечивающим достаточно малые расходы, является пористая вставка на выходе из баллона. 
«Системні технології» 3 (128) 2020 «System technologies»

Расчет хранения и расходования газа безусловно усложняется весьма малыми темпами опорожнения баллона, что вынуждает рассчитывать этот процесс на протяжении длительного времени, что затруднительно с вычислительной точки зрения. Как будут показано ниже, безразмерное время процесса расходования газа, входящее в обыкновенное дифференциальное уравнение, которое описывает опорожнение баллона, и безразмерное время процесса фильтрации газа через тонкую пористую вставку весьма разнятся по порядку величин. Налицо многомасштабный процесс, связанный со всеми специфическими вычислительными трудностями и особенностями, присущими таким процессам. Чтобы преодолеть упомянутые трудности, в настоящей работе использовался специально разработанный асимптотический алгоритм, позволяющий проводить расчет в «медленном времени» и тем самым заметно экономить вычислительные ресурсы.

Актуальность данной работы не вызывает ни малейшего сомнения в силу необходимости снабдить разрабатываемые космические летательные аппараты и установленные на них научно-исследовательские приборы и технические устройства надлежащим количеством газообразных веществ.

Анализ последних достижений и публикаций по тематике исследования. Термодинамика хранения газов в сжатом состоянии в специальных баллонах к настоящему времени достаточно хорошо изучена и отражена в известных монографиях [1-3], однако вопросы регулирования сверхмалых расходов газа в указанных книгах и других специализированных изданиях практически не отражены, хотя способ уменьшения расхода при помощи пористых вставок общеизвестен [4-7]. Что касается процессов фильтрации газа через пористые вставки и другие тонкие пористые тела, то общая теория таких течений содержится в многочисленных книгах по фильтрации, например [5-7], а частные математические модели фильтрационных течений рассматриваемого класса предложены в работе [8]. Вопросы асимптотических методов построения упрощенных математических моделей физических процессов отражены в общеизвестных монографиях по асимптотическому анализу [9-11]. 82 
«Системні технології» 3 (128) 2020 «System technologies»

Термодинамические свойства газообразных веществ для проведения расчетов могут быть найдены в справочном издании [12].

Цель работы. Основываясь на вышеизложенном, цель настоящей работы можно сформулировать следующим образом: разработка математической модели процессов хранения и расходования газообразных веществ в баллонах высокого давления и применение разработанного подхода к проблеме обеспечения инертными газами оборудования на борту космических аппаратов разного функционального назначения.

Основной материал исследования. Рассмотрим баллон объема $V$, первоначально заполненный некоторым сжатым газом с параметрами: масса $m_{0}$, молекулярная масса $\mu$, плотность $\rho_{0}=m_{0} / V$, температура $T_{0}$ и давление $p_{0}$. Соответственно, в процессе опорожнения актуальные параметры обозначим через $m, \rho, T$ и $p$. В дальнейшем при разработке физической модели будем полагать, что указанный газ относится к инертным газам, в противном случае необходимо провести дополнительный анализ, который, впрочем, качественно не изменит изложенной в дальнейшем расчетной схемы. Пусть из баллона газ истекает через пористую вставку. Не уменьшая общности, предположим, что вставка имеет постоянные пористость, коэффициент фильтрации и выполнена в форме кругового цилиндра (рис. 1).

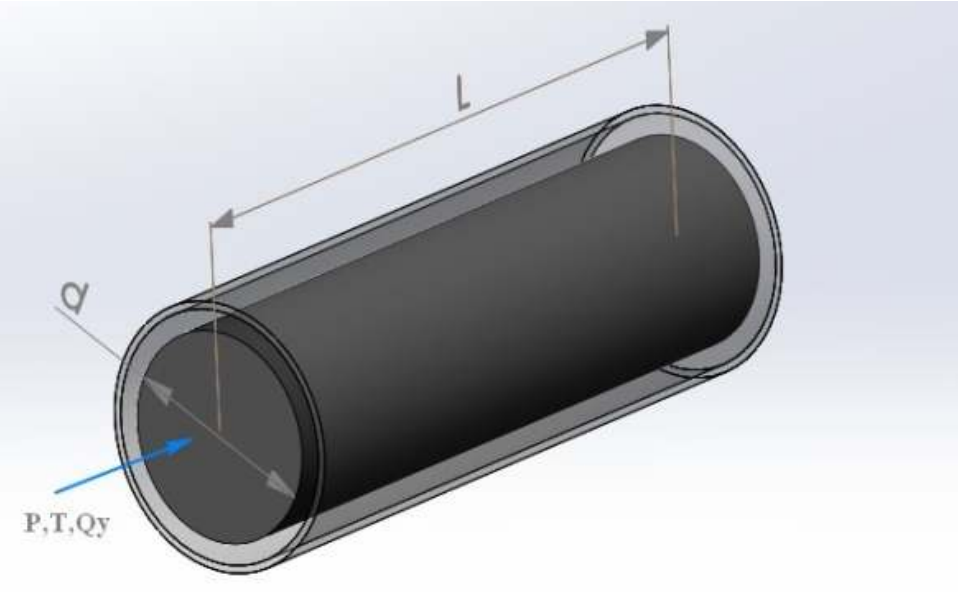

Рисунок 1 - Пористый элемент

Одноатомные инертные газы хорошо соответствуют уравнению состояния идеального газа [1] 
«Системні технології» 3 (128) 2020 «System technologies»

$$
p_{0} V_{0}=\frac{m_{0}}{\mu} R T_{0} \text { или } p V=\frac{m}{\mu} R T \text {, }
$$

где $R$ - универсальная газовая постоянная. Соотношения (1) иногда удобно записывать, используя плотность газа

$$
\frac{p_{0}}{\rho_{0}}=R^{*} T_{0} \text { или } \frac{p}{\rho}=R^{*} T,
$$

где $R^{*}$ - газовая постоянная данного газа.

Обозначив фильтрационный поток газа через пористую вставку через $q$ ( $q_{0}$ в начальный момент времени, соответственно), можно записать количественные соотношения для опорожнения газового баллона

$$
\begin{gathered}
\frac{d m}{d t}=q, \\
m(t=0)=m_{0},
\end{gathered}
$$

где $t$ - время.

Задача Коши (3), (4) может быть проинтегрирована численно любым из численных методов решения подобных задач [13], однако рассмотрим простейшие частные случаи аналитического интегрирования. Во-первых, случай $q=$ const, тогда

$$
m=m_{0}+q t .
$$

Здесь масса не возрастает с течением времени, поскольку при опорожнении баллона $q<0$. Во-вторых, случай $q(t)$, тогда

$$
m=m_{0}+\int_{t_{0}}^{t} q(t) d t .
$$

Наконец, случай зависимости расхода от массы газа в баллоне $q(m)$, тогда

$$
\int \frac{d m}{q(m)}=t+c,
$$

где постоянная с может быть найдена из условия (4). Отметим, что решение (5) является частным случаем решений (6) и (7), но выделено в отдельное предоставление из-за практической важности.

Для дальнейшего анализа предоставляется целесообразным обезразмерить задачу Коши (3), (4) следующим образом 
«Системні технологіï» 3 (128) 2020 «System technologies»

$$
\bar{m}=\frac{m}{m_{0}}, \quad \bar{q}=\frac{q}{q_{0}},
$$

тогда

$$
\begin{gathered}
\frac{d \bar{m}}{d \bar{t}}=\bar{q}, \\
\bar{m}(t=0)=1,
\end{gathered}
$$

где

$$
\bar{t}=\frac{t q_{0}}{m_{0}} .
$$

Однако

$$
\frac{q_{0}}{m_{0}}<<1 .
$$

Это условие длительной эксплуатации баллона для газоснабжения. Тогда время, определенное соотношением (11) можно назвать «медленным временем».

Прежде чем перейти к анализу фильтрационного течения в пористой вставке, целесообразным представляется сделать некоторые замечания относительно технологии газоснабжения из баллона. Управляющими параметрами процесса фильтрации могут быть только давление на внутренней и внешней сторонах пористой вставки. Если давление на внешней стороне определяется теплофизическими особенностями функционирования системы, потребляющей газ, то есть не может изменяться произвольным образом для изменения расхода газа, то давление внутри баллона может и должно изменяться с целью управления процессом газоснабжения. Изменение давления внутри баллона механическими способами (например, изменением $V$ в (1), (2)) труднореализуемо по техническим причинам, зато изменение температуры баллона ( $T$ в (1), (2)) может быть выполнено относительно легко, поэтому в дальнейшем будем полагать, что управление режимом фильтрации будет производиться только при помощи нагрева (охлаждения) баллона.

Дабы избежать путаницы в обозначениях, в уравнении фильтрации для давления будем использовать большую букву $P$, тогда, полагая, что фильтрационное течение описывается законом Дарси [4-6]:

$$
\vec{u}=-k \operatorname{grad} P,
$$

ISSN 1562-9945 (Print) 


\section{«Системні технології» 3 (128) 2020 «System technologies»}

где $\vec{u}$ - скорость фильтрационного течения, $k$ - коэффициент фильтрации, оператор grad понимается в традиционном смысле; можно получить следующее уравнение изотермической фильтрации газа [5]

$$
\frac{\partial P}{\partial t}=\frac{k}{2 \sigma} \Delta\left(P^{2}\right)
$$

где $\sigma$ - пористость среды, $\Delta$ - оператор Лапласа. Величина $\chi=\frac{k}{2 \sigma}$ зависит от структуры пористого материала. Уравнение (14) в рассматриваемом случае является одномерным по пространству, то есть

$$
\frac{\partial P}{\partial t}=\chi \frac{\partial^{2} P^{2}}{\partial x^{2}} .
$$

Обезразмерим уравнение (15)

$$
\frac{\partial P^{*}}{\partial t^{*}}=\frac{\partial^{2} P^{* 2}}{\partial x^{* 2}},
$$

где $P^{*}=P / p_{0}, x^{*}=x / L(L-$ длина пористой вставки $), t^{*}=\frac{t \chi p_{0}}{L^{2}}$. Однако, использование безразмерного времени $t^{*}$ для процесса опорожнения неудобно, а желательно перейти к масштабу времени $\bar{t}(11)$.

$$
\frac{\partial P^{*}}{\partial \bar{t}} \frac{\bar{t}}{t^{*}}=\frac{\partial^{2} P^{* 2}}{\partial x^{* 2}} \text {. }
$$

Обозначим

$$
\varepsilon=\frac{\bar{t}}{t^{*}}=\frac{\frac{t q_{0}}{m_{0}}}{\frac{t \chi p_{0}}{L^{2}}}=\frac{q_{0} L^{2}}{\chi m_{0} p_{0}} .
$$

Величина $\varepsilon$ в силу условия (12) является малой $(\varepsilon<<1)$. Уравнение (17) должно быть дополнено начальными и граничными условиями

$$
\begin{gathered}
P^{*}(t=0)=p_{0}, \\
P^{*}\left(x^{*}=0\right)=P, \\
P^{*}\left(x^{*}=1\right)=P_{\text {out }},
\end{gathered}
$$

где $P_{\text {out }}$ - известная функция времени (правда, как правило $P_{\text {out }}=$ const). 
«Системні технології» 3 (128) 2020 «System technologies»

Уравнение (17) включает малый параметр $\varepsilon$, применим к нему метод малого параметра, согласно которому решение отыскивается в виде

$$
P^{*}=P^{* 0}+\varepsilon P^{* 1}+\varepsilon P^{* 2}+\ldots .
$$

Подставляя разложение (22) в уравнение (17), группируя и приравнивая 0 суммы коэффициентов при одинаковых степенях $\varepsilon$, получим следующую последовательность дифференциальных уравнений:

$$
\begin{gathered}
\frac{\partial^{2}\left(P^{* 0}\right)^{2}}{\partial x^{*} 2}=0, \\
\frac{\partial^{2}\left(P^{* 1}\right)^{2}}{\partial x^{* 2}}=\frac{\partial P^{* 0}}{\partial \bar{t}},
\end{gathered}
$$

для которых с учетом граничных условий (20), (21) легко формулируются соответствующие краевые задачи.

При достаточно медленных процессах опорожнения можно ограничиться рассмотрением только краевой задачи для уравнения (23). При этом начальные условия для такой краевой задачи не нужны, а граничные условия (20), (21) могут быть использованы без изменений. Уравнение (23) имеет очевидный первый интеграл

$$
\left(P^{* 0}\right)^{2}=a x^{*}+b \text {. }
$$

С учетом условий (20), (21)

$$
b=p^{2}, \quad a=P_{\text {out }}^{2}-p^{2} .
$$

Таким образом, задачу фильтрации газа в нулевом приближении можно считать решенной. В полученном решении $P^{*}$ и $P_{\text {out }}$ могут зависеть от времени, но если зависимость от времени величины $P_{\text {out }}$ задается в граничном условии (21), то зависимость от времени величины $P^{*}$ определяется ходом протекания процесса опорожнения баллона и нагревом (охлаждением) последнего. Возвращаясь к размерным переменным, запишем формулу для величины расхода

$$
q=\rho S u
$$

где $S$ - площадь поперечного сечения пористой вставки, $u$ - скорость фильтрационного течения, определяемая законом Дарси (13). 
«Системні технології» 3 (128) 2020 «System technologies»

С прикладной точки зрения и в соответствии с целями и задачами настоящей работы наибольший интерес представляет случай $q=$ const, описываемый решением (5). Разделив обе стороны равенства (5) на объем $V$, получим закон изменения плотности при постоянном расходе

$$
\rho=\rho_{0}+\frac{q}{V} t
$$

Из условия постоянства расхода (27) и закона изменения плотности (28) следует закон изменения скорости (площадь поперечного сечения $S$ постоянна)

$$
u=\frac{q}{S\left(\rho_{0}+q t\right)}
$$

но по закону Дарси (13) и в силу решения (25), запишем в размерном виде следующие соотношения:

$$
\begin{gathered}
\frac{\partial P}{\partial x}=\frac{P_{\text {out }}^{2}-p^{2}}{2 L p}, \\
-k \frac{P_{\text {out }}^{2}-p^{2}}{2 L p}=\frac{q}{S\left(\rho_{0}+q t\right)} .
\end{gathered}
$$

Полученное уравнение (31) можно рассматривать как квадратное уравнение для определения давления в баллоне

$$
p^{2}-\frac{2 q L}{S\left(\rho_{0}+\frac{q t}{V}\right)} p-P_{\text {out }}^{2}=0 \text {. }
$$

Понятно, что при решении уравнения (32) из двух его корней (а они всегда существуют, поскольку дискриминант этого уравнения положителен) следует выбирать положительный корень. В силу соотношений (2) температура газа в баллоне связана с давлением следующим соотношением

$$
T=\frac{p}{R \rho}
$$

а это означает, что для поддержания постоянного значения расхода газа, к нему должно быть подведено количество теплоты

$$
Q=m c_{v} \frac{\partial T}{\partial t}
$$

где $c_{v}$ - теплоемкость газа при постоянном объеме. 
«Системні технології» 3 (128) 2020 «System technologies»

Кроме того, к самому баллону должно быть подведено количество теплоты, компенсирующее его теплообмен с окружающей средой, однако анализ указанного процесса теплообмена далеко выходит за рамки настоящей работы, поскольку является частью общего расчета теплового режима космического летательного аппарата.

Замечание. При непостоянном заданном расходе газа $q$ и переменном внешнем давлении $P_{\text {out }}$, которое также полагается заданным, схема расчета принципиально остается такой же самой, однако некоторые процедуры расчета усложняются. Поскольку побудительным мотивом варьирования указанных функций являются релевантные процедуры функционирования летательного космического аппарата, здесь этот вопрос рассматриваться не будет.

Анализ полученных результатов. В настоящей работе предложена математическая модель и построено приближенное аналитическое решение (в нулевом приближении) для процесса истечения газа из баллона, закрытого пористой вставкой. Точность предложенной математической модели определяется следующими моментами: правомерностью использования уравнения состояния идеального газа, что подтверждено обширным опытом применения такого подхода для расчета термодинамических процессов в инертных газах при умеренных температурах и не сверхвысоких давлениях; правомерностью использования модели изотермической фильтрации газа, что также подтверждается обширным опытом успешного проведения подобного рода расчетов в практических целях; точностью построенной асимптотической математической модели фильтрации, как можно показать, погрешность данной модели имеет порядок $\varepsilon$, а по условиям задачи $\varepsilon$ является достаточно малой величиной. Все остальные этапы решения были проделаны аналитически и не вносили дополнительной погрешности.

Несмотря на то, что в настоящей работе не рассматривалась схема нагрева (охлаждения) баллона, можно рекомендовать поместить нагревательные элементы внутрь баллона, чтобы уменьшить теплопотери в окружающее пространство. Случай, когда для поддержания заданного режима опорожнения требуется охлаждение баллона, с конструктивно- 


\section{«Системні технології» 3 (128) 2020 «System technologies»}

технологической точки зрения является нежелательным, хотя предложенная математическая модель и соответствующий ей метод расчета вполне допускают случай охлаждения баллона. Вопросы управления процессом опорожнения баллона со сжатым газом через пористую вставку при помощи нагрева или охлаждения баллона также могут быть поставлены в рамках предложенной математической модели. Однако в настоящей работе эти вопросы не рассматривались, поскольку для анализа этого процесса необходимо определить тепловой режим отсека, в котором установлен баллон.

Выводы и анализ перспектив дальнейших исследований. Применение асимптотического подхода позволило построить достаточно простую приближенную асимптотическую математическую модель процесса опорожнения газового баллона с экстремально малым расходом газа. В нулевом приближении построенная модель допускает элементарное аналитическое решение, что выгодно отличает ее от альтернативных подходов, требующих численного анализа.

Обеспечение функционирования систем космических летательных аппаратов во время длительных полетов зачастую требует нетрадиционных технических решений. Одним из таких решений является использование пористых вставок в качестве дозатора в системе газоснабжения. В настоящей работе показано, что расчет указанных типов систем газоснабжения может быть проведен при помощи несложных аналитических методов. Полученное решение представляет интерес не только для развития ракетно-космической техники, но для других областей техники и технологий, где предусмотрено хранение и использование сжатых газов.

Перспективы дальнейших исследований совершенно очевидны и заключаются во включении разработанной расчетной схемы в качестве составной части в общую расчетную схему космического летательного аппарата, что обеспечит возможности комплексного анализа рассматриваемых процессов совместно с процессами, функционально с ними связанными. 


\section{«Системні технології» 3 (128) 2020 «System technologies»}

\section{ЛИТЕРАТУРА / ЛІТЕРИРУРА}

1. Беляев Н. М. Термодинамика / Н. М. Беляев. - К.: Вища школа, 1987. 382 c.

2. Павловский В. А. Введение в термодинамику реальных газов: Монография ФГУП «Крыловский государственный научный центр» / В. А. Павловский. - СПб., 2013. - 230 с.

3. Павловский В. А. Моделирование динамики заполнения резервуара реальным газом / В. А. Павловский, А. Л. Чистов. - СПб., 2013.

4. Пилатовский $\quad$ В. П. Основы гидромеханики тонкого пласта / В. П. Пилатовский. - М.: Недра, 1966. - 317 с.

5. Барренблатт Г. И. Теория нестационарной фильтрации жидкости и газа / Г. И. Барренблатт, В. М. Ентов, В. М. Рыжик. - М.: Недра, 1972. -288 с. 6. Коллинз Р. Течения жидкостей через пористые материалы / Р. Коллинз. - М.: Мир, 1964. - 352 с.

7. Петрянов И. В. Волокнистые фильтрующие материалы ФП / И. В. Петрянов, В. И. Козлов, П. И. Басманов, Б. И. Огородников. - М.: Химия, 1968. - 79 с.

8. Бразалук Ю. В. Асимптотические математические модели фильтрации газа через тонкую стенку. Часть 1. Постановка проблемы, изотермический случай / Ю. В. Бразалук, А. И. Губин, А. В. Давыдова, Д. В. Евдокимов, М. А. Стояновский, Р. А. Шульга // Системи проектування та аналіз характеристик аерокосмічної техніки. - Том 24. - Д.: Ліра, 2018. - C. 15-40.

9. Найфэ А. Х. Методы возмущений / А. Х. Найфэ. - М.: Мир, 1976. - 456 с. 10. Коул Д. Д. Методы возмущений в прикладной математике / Д. Д. Коул. - М.: Мир, 1972. - 276 с.

11. Ван-Дайк М. Методы возмущений в механике жидкости / М. ВанДайк. - М: Мир, 1967. - 296 с.

12. Гурвич Л. В. Термодинамические свойства индивидуальных веществ: справочное издание в 4-х т. / Л. В. Гурвич, И. В. Вейц, В. А. Медведев и др. - 3-е изд., перераб. и расширен. - Т. І, кн. 1. - М.: Наука, 1978. - 496 с. 13. Демидович Б. П. Численные методы анализа / Б. П. Демидович, И. А. Марон, Э. З. Шувалова. - М.: Наука, 1967. - 368 с. 


\section{«Системні технології» 3 (128) 2020 «System technologies»}

\section{REFERENCES}

1. Belyaev N. M. Termodinamika / N. M. Belyaev. - K.: Vischa shkola, 1987. $382 \mathrm{~s}$.

2. Pavlovskiy V. A. Vvedenie v termodinamiku realnyih gazov: Monografiya FGUP «Kryilovskiy gosudarstvennyiy nauchnyiy tsentr» / V. A. Pavlovskiy. SPb., 2013. - $230 \mathrm{~s}$.

3. Pavlovskiy V. A. Modelirovanie dinamiki zapolneniya rezervuara realnyim gazom / V. A. Pavlovskiy, A. L. Chistov. - SPb., 2013.

4. Pilatovskiy V. P. Osnovyi gidromehaniki tonkogo plasta / V.P. Pilatovskiy. M.: Nedra, 1966. - 317 s.

5. Barrenblatt G. I. Teoriya nestatsionarnoy filtratsii zhidkosti i gaza /

G. I. Barrenblatt, V. M. Entov, V. M. Ryizhik. - M.: Nedra, 1972. -288 s.

6. Kollinz R. Techeniya zhidkostey cherez poristyie materialyi / R. Kollinz. M.: Mir, 1964. - $352 \mathrm{~s}$.

7. Petryanov I. V. Voloknistyie filtruyuschie materialyi FP / I. V. Petryanov, V. I. Kozlov, P. I. Basmanov, B. I. Ogorodnikov. - M.: Himiya, 1968. - 79 s.

8. Brazaluk Iu. V. Asimptoticheskie matematicheskie modeli filtratsii gaza cherez tonkuyu stenku. Chast 1 . Postanovka problemyi, izotermicheskiy sluchay / Iu. V. Brazaluk, O. I. Gubin., A. V. Davydova, D. V. Yevdokymov, M. A. Stoyanovskiy, R. O. Shulha // Systemy proektuvannia ta analiz kharakterystyk aerokosmichnoi tekhniky. - Tom 24. - D.: Lira, 2018. - S. 15-40.

9. Nayfe A. H. Metodyi vozmuscheniy / A. H. Nayfe. - M.: Mir, 1976. - 456 s.

10. Koul D. D. Metodyi vozmuscheniy v prikladnoy matematike / D. D. Koul. M.: Mir, 1972. - $276 \mathrm{~s}$.

11. Van-Dayk M. Metodyi vozmuscheniy v mehanike zhidkosti / M. VanDayk. - M: Mir, 1967. - 296 s.

12. Gurvich L. V. Termodinamicheskie svoystva individualnyih veschestv: spravochnoe izdanie v 4-h t. / L. V. Gurvich, I. V. Veyts, V. A. Medvedev i dr. 3-e izd., pererab. i rasshiren. - T. I, kn. 1. - M.: Nauka, 1978. - 496 s.

13. Demidovich B. P. Chislennyie metodyi analiza / B. P. Demidovich, I. A. Maron, E. Z. Shuvalova. - M.: Nauka, 1967. - 368 s.

Received 17.02.2020. Accepted 20.02.2020.

Математическое моделирование баллонных систем хранения и регулирования расхода газа

В работе рассмотрены процессы истечения газа через пористую вставку из баллона высокого давления, используемого для газоснабжения космического летательного аппарата в длительном полете. Для описания процесса фильтрации была использована 92 


\section{«Системні технологіï» 3 (128) 2020 «System technologies»}

математическая модель изотермической фильтрации газа. Применение асимптотического подхода позволило свести описание процесса фильтрации к краевой задаче для обыкновенного дифференциального уравнения второго порядка, которую удалось решить аналитически. Показано, что в качестве управляющих параметров процесса истечения газа можно использовать температуру газа внутри баллона или давление газа на выходе пористой вставки. Результаты работы могут быть рекомендованы для использования в ракетно-космической технике и других областях, связанных с хранением сжатого газа.

Mathematical modeling of balloon systems for storage and rate control of gas

Modern techniques and technologies often require constant or controlled gas supply for their functionality. Especially difficult this problem appears in transport systems, where a gas source must be transported by the same system, for example, in aviation vehicles and space vehicles. The long-time flight usually takes place in the last case. Processes of gas outflow from high-pressure balloon using for gas-supply of space vehicle in long-time flow are considered in the work. To provide an enough small discharge, a porous insertion is applied as a rate control tool in the balloon. It is necessary to note, that mathematical models of such constructions is not developed yet, nevertheless huge amount of investigations concerning filtration flows. It is assumed that the inertial gases are used, which are enough good satisfied to ideal gas state equation. The well-known mathematical model of isothermal gas filtration is used to describe a filtration process. Taking into account slow outflow, it is managed to apply an asymptotic approach for analysis of the mentioned filtration model. Such trick is completely necessary to overcome difficulties of multiscale problem solution, arising due to smallness of filtration flow rate. As a rule, the obtained asymptotic sequence of problems can be restricted by only zero-th approximation because of the mentioned smallness of filtration flow rate and thus smallness of the constructed small parameter used in asymptotic expansion. As a result, a description of filtration process is reduced to boundary-value problem for second order ordinary differential equation. It is managed to integrate the obtained boundary-value problem in quadrature and to reduce an initial problem to quadratic equation with respect to the pressure in the balloon. It is shown, that the temperature inside the balloon and gas pressure on the porous insertion outlet can be used as control parameters for the process of gas outflow from high-pressure balloon through porous insertion. Thus a new asymptotic approach is developed for specific kind of strongly nonlinear problems. The recommendations concerning heating and cooling of the balloon are formulated. The results of the work can be recommended for using in space-missile technique and other field, connected with pressured gas storage.

Бразалук Юлия Владимирова - доцент кафедры аэрогидромеханики и энергомассопереноса, Днепровский национальный университет имени Олеся Гончара.

Губин Александр Игоревич - доцент кафедры аэрогидромеханики и энергомассопереноса, Днепровский национальный университет имени Олеся Гончара.

Давыдова Анжелика Владимировна - доцент кафедры космических информационных технологий, Днепровский национальный университет имени Олеся Гончара. 


\section{«Системні технології» 3 (128) 2020 «System technologies»}

Дерий Вероника Станиславовна - аспирантка кафедры аэрогидромеханики и энергомассопереноса, Днепровский национальный университет имени Олеся Гончара.

Евдокимов Дмитрий Васильевич - младший научный сотрудник кафедры теоретической и компьютерной механики, Днепровский национальный университет имени Олеся Гончара.

Малая Юлия Анатольевна - доцент кафедры компьютерных наук и инженерии программного обеспечения, Университет таможенного дела и финансов.

Бразалук Юлія Володимирівна - доцент кафедри аерогідромеханіки та енергомасопереносу, Дніпровський національний університет імені Олеся Гончара.

Губін Олександр Ігорович - доцент кафедри аерогідромеханіки та енергомасопереносу, Дніпровський національний університет імені Олеся Гончара.

Давидова Анжеліка Володимирівна - доцент кафедри космічних інформаційних технологій, Дніпровський національний університет імені Олеся Гончара.

Дерій Вероніка Станіславівна - аспірантка кафедри аерогідромеханіки та енергомасопереносу, Дніпровський національний університет імені Олеся Гончара.

Євдокимов Дмитро Васильович - молодший науковий співробітник кафедри теоретичної та комп'ютерної механіки, Дніпровський національний університет імені Олеся Гончара.

Мала Юлія Анатоліївна - доцент кафедри комп’ютерних наук та інженерії програмного забезпечення, Університет митної справи та фінансів.

Brazaluk Iuliia - associated professor of aerohydromechanics and energy and mass transfer chair, Oles Honchar Dnipro National University.

Gubin Oleksandr - associated professor of aerohydromechanics and energy and mass transfer chair, Oles Honchar Dnipro National University.

Davydova Angelika - associated professor of space information technology chair, Oles Honchar Dnipro National University.

Deriy Veronika - post-graduate student of aerohydromechanics and energy and mass transfer chair, Oles Honchar Dnipro National University.

Yevdokymov Dmytro - junior researcher of theoretical and computer mechanics chair, Oles Honchar Dnipro National University.

Mala Yuliia - associated professor of computer science and software engineering chair, University of Customs and Finance. 